\title{
Enriched cereal bars are more effective in increasing plasma quercetin compared with quercetin from powder-filled hard capsules
}

\author{
Sarah Egert ${ }^{1,2 *}$, Siegfried Wolffram ${ }^{3}$, Beate Schulze ${ }^{4}$, Peter Langguth ${ }^{5}$, Eva Maria Hubbermann ${ }^{4}$, \\ Karin Schwarz ${ }^{4}$, Berit Adolphi ${ }^{6}$, Anja Bosy-Westphal ${ }^{2}$, Gerald Rimbach ${ }^{7}$ and Manfred James Müller ${ }^{2}$ \\ ${ }^{1}$ Department of Nutrition and Food Science, Nutritional Physiology, University of Bonn, Endenicher Allee 11-13, \\ 53115 Bonn, Germany \\ ${ }^{2}$ Department of Human Nutrition, Institute of Human Nutrition and Food Science, Christian-Albrechts-University Kiel, Kiel, \\ Germany \\ ${ }^{3}$ Institute of Animal Nutrition and Physiology, Christian-Albrechts-University Kiel, Kiel, Germany \\ ${ }^{4}$ Department of Food Technology, Institute of Human Nutrition and Food Science, Christian-Albrechts-University Kiel, \\ Kiel, Germany \\ ${ }^{5}$ Department of Biopharmaceutics and Pharmaceutical Technology, Institute of Pharmacy, Johannes Gutenberg-University, \\ ${ }^{6}$ Schwartauer-Werke GmbH \& Co. KGaA, Bad Schwartau, Germany \\ ${ }^{7}$ Department of Food Science, Institute of Human Nutrition and Food Science, Christian-Albrechts-University Kiel, Kiel, \\ Germany
}

ᄃ Mainz, Germany

(Received 9 February 2011 - Revised 10 May 2011 - Accepted 13 May 2011 - First published online 20 July 2011)

\section{Abstract}

The flavonol quercetin, is one of the major flavonoids found in edible plants. The bioavailability of quercetin in humans may be influenced by the food matrix in which it is consumed as well as by its chemical and physical form. The objective of the present study was to investigate the biokinetics of quercetin from quercetin-enriched cereal bars and quercetin powder-filled hard capsules. In a randomised, single-blinded, diet-controlled cross-over study, six healthy women aged 22-28 years took a single oral dose of approximately 130 mg quercetin equivalents from either quercetin-enriched cereal bars (containing 93.3\% quercetin aglycone plus $6 \cdot 7 \%$ quercetin-4'-glucoside) or quercetin powder-filled hard capsules (100\% quercetin aglycone). Blood samples were drawn before and after quercetin administration over a $24 \mathrm{~h}$ period. The concentrations of quercetin and its monomethylated derivatives, isorhamnetin ( $3^{\prime}$-O-methyl quercetin) and tamarixetin (4'-O-methyl quercetin), were measured by HPLC with fluorescence detection after plasma enzymatic treatment. The systemic availability as determined by comparing the plasma concentration-time curves of quercetin was found to be five times and the $c_{\text {max }}$ values six times higher after ingestion of $130 \mathrm{mg}$ quercetin by quercetin-enriched cereal bars than after ingestion by quercetin capsules. In contrast, $t_{\max }$ did not differ significantly between the two treatments. The $c_{\max }$ values for isorhamnetin and tamarixetin were four and nine times higher after ingestion of quercetin by quercetin-enriched cereal bars than after ingestion by quercetin capsules. In conclusion, quercetin from quercetin-enriched cereal bars is significantly more bioavailable than from quercetin powder-filled hard capsules.

\section{Key words: Quercetin: Bioavailability: Onions: Human studies}

Quercetin (3, 3', $4^{\prime}, 5,7$-pentahydroxyflavone) is one of the major flavonoids, ubiquitously distributed in edible plants ${ }^{(1)}$. Epidemiological studies, together with data from animal models and some clinical trials, may suggest a role of flavonoids in general and of quercetin in particular in the prevention of CVD and other age-related chronic diseases ${ }^{(2)}$. In plasma, quercetin is found in the form of glucuronidated, sulphated and methylated derivatives ${ }^{(3)}$. Rich sources of dietary quercetin are onions, unpeeled apples, berries, citrus fruits, tea (Camellia sinensis) and red wine ${ }^{(1)}$. Daily dietary intake of the flavonol quercetin varies considerably. In Western populations, crude estimates of mean dietary intake appear to be $10-100 \mathrm{mg} / \mathrm{d}^{(1,4)}$. In some countries, quercetin is available as a dietary supplement with daily doses of 200-1200 mg quercetin (manufacturers' recommendations). In addition, quercetin may be used as a nutraceutical for functional foods within a concentration range of $0.008-$ $0.5 \%$ or $10-125 \mathrm{mg} /$ serving $^{(5)}$. Rather high concentrations of

Abbreviations: AUC, area under plasma concentration-time curve; $c_{\max }$, maximum plasma concentration; $t_{\text {max }}$, time between oral administration of quercetin and the appearance of $c_{\max }$.

*Corresponding author: S. Egert, fax +49 228 733217, email s.egert@uni-bonn.de 
quercetin in foods can be obtained through the addition of pure quercetin, but probably also by the enrichment of foods with sources naturally rich in quercetin.

A prerequisite for the physiological effects of quercetin is its bioavailability. A considerable number of studies have dealt with determining the bioavailability of quercetin in human subjects ${ }^{(3)}$. It has been reported that isolated quercetin glucosides are absorbed better than quercetin administered in non-glucosidic forms, with no difference between the $3^{\prime}$-O-glucoside and $4^{\prime}$-O-glucoside ${ }^{(6)}$. Furthermore, Hollman et $a l .{ }^{(7)}$ showed in healthy ileostomy volunteers that quercetin absorption was approximately two times higher when it was ingested as quercetin glucosides originating from fried onions compared with quercetin aglycone administered as a pure substance (quercetin dihydrate) in capsules. In contrast, Wiczkowski et $a l^{(8)}$ found that when provided along with natural sources (e.g. shallot preparations), quercetin aglycone was significantly more bioavailable than its glucosides in human subjects.

Onion skins are rich sources of quercetin aglycone ${ }^{(8)}$ and may be suitable for the enrichment of several foods with quercetin. However, at present it is not known to what extent quercetin from foods enriched with onion skins or with extracts from onion skins is absorbed and whether food components or the matrix affects its bioavailability.

The present pilot study was designed to investigate in healthy normal-weight volunteers the systemic availability of quercetin from cereal bars enriched with quercetin derived from onion skin extract and in comparison from pure quercetin powder-filled hard capsules, consumed in combination with quercetin-free cereal bars. A dosage of approximately $130 \mathrm{mg}$ quercetin was chosen, since it has been shown to lower resting systolic blood pressure, pulse pressure and plasma concentrations of oxidised LDL in our previous placebocontrolled cross-over study in overweight patients with a high CVD risk phenotype ${ }^{(9,10)}$. With both treatments, cereal bars and capsules, quercetin was administered as quercetin aglycone. In addition, the food matrix with which quercetin was ingested was the same; only the vehicle that contained the quercetin aglycone differed (cereal bar $v$. capsule).

\section{Subjects and methods}

\section{Subjects}

A total of six non-smoking German women aged 22-28 years participated in the present study. They were in good health as determined by a basic examination (body weight and height, blood pressure/pulse, medical anamneses and blood analyses; baseline characteristics are presented in Table 1). BMI ranged between 20 and $23 \mathrm{~kg} / \mathrm{m}^{2}$. Exclusion criteria were overweight, metabolic and endocrine diseases, malabsorption syndromes, smoking, pregnancy/lactation, alcohol abuse, use of dietary supplements or any form of medication (with the exception of oral contraceptives, which were taken by all women). All subjects were asked to maintain their regular lifestyles and usual extent of physical activities throughout the study. The study was conducted according to the guidelines laid down in the Declaration of Helsinki, and all procedures involving human subjects were approved by the ethics committee of the Medical Faculty of the Christian-Albrechts-University of Kiel, Germany. Written informed consent was obtained from all subjects.

\section{Study design}

Run-in phase. The participants refrained from quercetin-rich food for 1 week. For this purpose, a list of food items rich in quercetin (e.g. unpeeled apples, grape products, fruit juice, citrus fruits, berries, onions, broccoli, French beans, kale, tea and red wine) was given to each participant, and they were instructed to avoid such products. Compliance with these dietary restrictions was controlled by a self-completed standardised 3d dietary record. No deviation from the low-quercetin diet occurred as shown by the records. Accordingly, the concentrations of quercetin were very low (0.08 (sem 0.01) $\mu \mathrm{mol} / \mathrm{l})$ in fasting plasma samples taken at baseline (see later).

\section{Treatment period}

The study was conducted in a single-blinded (blind participants), diet-controlled cross-over design. The six women were randomly assigned to treatment group $\mathrm{A}$ or $\mathrm{B}$ ( $n 3$ and 3, respectively). Group A ingested two quercetin-enriched cereal bars (approximately $130 \mathrm{mg}$ quercetin equivalents) plus six placebo capsules with a glass of water $(200 \mathrm{ml})$; group B ingested two quercetin-free cereal bars ('placebo cereals bars') plus six quercetin capsules (approximately $130 \mathrm{mg}$ quercetin equivalents) with $200 \mathrm{ml}$ water during the first period of the treatment. After a break of 2 weeks (including a 1-week run-in period), group A ingested two placebo cereals bars plus six quercetin capsules during the second treatment and group B consumed two quercetin-enriched cereal bars plus six placebo capsules. The products were given in the morning after a $12 \mathrm{~h}$ overnight fast.

Table 1. Baseline characteristics of the participants (Mean values with their standard errors, $n 6$ )

\begin{tabular}{lcc}
\hline & \multicolumn{2}{c}{ Women } \\
\cline { 2 - 3 } Variable & Mean & SEM \\
\hline Age (years) & 25.7 & 0.93 \\
Body height $(\mathrm{m})$ & 1.66 & 0.03 \\
Body weight $(\mathrm{kg})$ & 58.5 & 1.8 \\
BMI $\left(\mathrm{kg} / \mathrm{m}^{2}\right)$ & 21.3 & 0.6 \\
Fat mass $(\%)$ & 29.3 & 2.9 \\
Fasting serum total cholesterol $(\mathrm{mmol} / \mathrm{l})$ & 4.90 & 0.28 \\
Fasting serum LDL-cholesterol $(\mathrm{mmol} / \mathrm{l})$ & 2.24 & 0.13 \\
Fasting serum HDL-cholesterol $(\mathrm{mmol} / \mathrm{l})$ & 2.19 & 0.22 \\
Fasting serum TAG (mmol/l) & 1.00 & 0.11 \\
Fasting plasma glucose $(\mathrm{mmol} / \mathrm{l})$ & 4.48 & 0.10 \\
Haematocrit $(\%)$ & 37.1 & 0.65 \\
Blood Hb (g/l) & 127.5 & 2.5 \\
Fasting plasma quercetin $(\mu \mathrm{mol} / \mathrm{l})$ & 0.08 & 0.01 \\
Systolic blood pressure $(\mathrm{mmHg})$ & 113 & 2.1 \\
Diastolic blood pressure $(\mathrm{mmHg})$ & 73 & 1.8 \\
\hline
\end{tabular}


A standardised breakfast (white bread, butter and cheese and $200 \mathrm{ml}$ water) was offered $120 \mathrm{~min}$ after the quercetin bolus. Lunch (pasta, cheese sauce, ice cream and $200 \mathrm{ml}$ water) was served $240 \mathrm{~min}$ after quercetin administration, and dinner (white bread, butter, cheese, yogurt and water) was served at $600 \mathrm{~min}$ after quercetin administration. All foods were weighed to the nearest gram, contained only minor amounts of flavonoids and were provided at the study site. The participants were only allowed to drink water. Food and water intake was documented in a dietary record.

Blood samples were collected before (baseline) quercetin administration and 30, 60, 90, 120, 180, 240, 300, 360, 420, 480 and $1440 \mathrm{~min}$ after the dose. Blood was obtained by an indwelling cannula for samples up to $8 \mathrm{~h}$ and thereafter by venipuncture.

\section{Study products}

The study products used are not commercially available and were specifically made for the present biokinetic study. The quercetin and placebo capsules were prepared in a Good Manufacturing Practice (GMP)-certificated facility (Department of Biopharmaceutics and Pharmaceutical Technology, Johannes Gutenberg-University Mainz, Germany). The hard gelatine capsules were filled with $25 \mathrm{mg}$ quercetin dihydrate powder (Voigt Global Distribution, Inc., Lawrence, KS, USA), mannitol and the flow-regulating excipient silicium dioxide. The quercetin content of the quercetin capsules was constant for a shelf-life of 3 years. The placebo capsules contained only mannitol and silicium dioxide.

The cereal bars were produced by Schwartauer Werke (Bad Schwartau, Germany). The main ingredients of the formulations were milk chocolate, glucose-fructose syrup, popped cereals (meal), almonds and cereal flakes. Enrichment with quercetin was achieved by mixing the onion skin extract (Rudolf Wild GmbH \& Company KG, Heidelberg-Eppelheim, Germany) with almonds, cereal flakes and popped cereals before adding the viscous sugar binder. Finally, the cereal bars were partly coated with milk chocolate.

The quercetin content of the cereal bars was constant for a stated shelf-life of 12 months. Placebo cereal bars were prepared and packaged in an identical form to the enriched cereal bars to allow the blind protocol. The nutritional content of two cereal bars $(2 \times 25 \mathrm{~g})$ was $940 \mathrm{~kJ}$ energy, $3.8 \mathrm{~g}$ protein, $29.4 \mathrm{~g}$ carbohydrate, $15.6 \mathrm{~g}$ sugar, $9 \mathrm{~g}$ fat, $4 \mathrm{~g}$ SFA, $2.2 \mathrm{~g}$ dietary fibre and $0 \cdot 1 \mathrm{~g} \mathrm{Na}$. Quercetin-enriched cereal bars and placebo cereal bars were comparable with respect to appearance, texture, taste, acceptability and palatability.

The particle size $\mathrm{X}_{50}$ of quercetin dihydrate (capsules) was $11.9 \mu \mathrm{m}$. The $\mathrm{X}_{50}$ of the onion skin extract (cereal bars) averaged $22.5 \mu \mathrm{m}$. For the determination of particle size, a laser diffraction sensor (Helos; Sympatec GmbH, ClausthalZellerfeld, Germany) equipped with a cuvette was used. For the measurement of the particle size, an aliquot of the powder was dispersed in an inert oil (MCT (medium-chain TAG) oil; Gustav Hees Oleochemische Erzeugnisse GmbH, Stuttgart, Germany) until an optical density of 5\% was obtained. Analyses were performed in triplicate.

\section{HPLC analyses of quercetin in cereal bars and capsules}

The quercetin content of the capsules and cereal bars was determined by HPLC-diode-array detection analysis. For the determination of the content of the cereal bars, approximately $1 \mathrm{~g}$ of the homogenised sample was weighed into an extraction tube and $10 \mathrm{ml}$ of a mixture of $80 \%$ acetone and $20 \%$ aqueous $0 \cdot 1 \%$ citric acid solution were added. Extraction was performed using ultrasonication. The samples were centrifuged $(8750 \mathrm{~g}$, $3 \mathrm{~min}, 20^{\circ} \mathrm{C}$ ) and the supernatant was collected. The extraction was repeated three times using $5 \mathrm{ml}$ of the extraction solvent for each extraction step. The supernatants were combined and filled to a total volume of $25 \mathrm{ml}$. For the capsules, the content of one capsule was dissolved in $100 \mathrm{ml}$ of a mixture of $80 \%$ acetone and $20 \%$ aqueous $0 \cdot 1 \%$ citric acid solution. Before HPLC analysis, the samples were filtered through a paper filter. Analyses were carried out in duplicate.

Immediately after extraction, the extracts were analysed by HPLC-diode-array detection on an Agilent HP 1100 instrument (Agilent Technologies, Waldbronn, Germany) equipped with a Nucleodur Sphinx RP-C18 column $(125 \times 4 \mathrm{~mm} ; 5 \mu \mathrm{m}$, Machery \& Nagel, Düren, Germany) using $0.5 \%$ aqueous formic acid (solvent A) and 100\% acetonitrile (solvent B) at a flow rate of $1 \mathrm{ml} / \mathrm{min}$ with gradient elution: $0-15 \mathrm{~min}, 10 \% \mathrm{~B}$; 15-20 min, $10-28.5 \% \mathrm{~B}$; 20-25 min, $28 \cdot 5-34 \% \mathrm{~B}$; 25-30 min, $34-44 \% \mathrm{~B} ; 30-34 \mathrm{~min}, 44-80 \% \mathrm{~B}$ and $34-38 \mathrm{~min}, 80-10 \%$ B. Analytes were detected at $365 \mathrm{~nm}$ and quantified using quercetin aglycone for calibration.

In addition, the declared quercetin content of the capsules of $22.03 \mathrm{mg}$ quercetin aglycone (anhydrous) was verified by UV spectroscopy using a sample size of twenty quercetin capsules. The content of each capsule was dissolved in $35 \mathrm{ml}$ acetone. The solution was transferred to a $100 \mathrm{ml}$ volumetric flask and filled to volume with demineralised water, and $50 \mu \mathrm{l}$ of this solution was added to an acetone-water mixture (35:65) and absorbance was measured at $374 \mathrm{~nm}$.

\section{Dietary analysis}

Dietary records were analysed using the computer-based nutrient calculation program EBISpro (University of Hohenheim, Stuttgart, Germany), based on the German Nutrient Database Bundeslebensmittelschlüssel (Max Rubner-Institute, Karlsruhe, Germany).

\section{Nutritional status}

Body height was measured to the nearest $0.5 \mathrm{~cm}$ with a stadiometer during the basic examination. Body composition (fat mass and fat-free mass) and body weight were determined on each treatment day after an overnight fast by air displacement plethysmography using the BOD POD Body Composition System (Life Measurements Instruments, Concord, CA, USA) ${ }^{(11)}$.

\section{Blood sampling, processing and analysis}

For the determination of plasma concentrations of quercetin and its metabolites, blood was drawn into tubes containing 
lithium heparin (Sarstedt, Nümbrecht, Germany). After each sampling, blood was immediately centrifuged $(2000 \mathrm{~g}$, $15 \mathrm{~min}, 4^{\circ} \mathrm{C}$ ) and the plasma was stored at $-75^{\circ} \mathrm{C}$ until analysis. All samples from one participant were measured within one analytical run. The analyses were conducted without the knowledge of the treatment groups.

On each treatment, additional fasting blood was taken to determine serum total cholesterol, LDL-cholesterol, HDLcholesterol and TAG concentrations as well as plasma glucose concentrations, liver and kidney function markers in serum and haematological markers in blood.

Fasting blood concentrations of glucose, lipids as well as liver and kidney function markers were measured on a VITROS 950 Chemistry System autoanalyser (Ortho-Clinical Diagnostics, Neckargemünd, Germany) with the manufacturer's assay kits, quality controls and reagents. Haematological parameters (leucocyte count, erythrocyte count, platelet count, $\mathrm{Hb}$ concentration, haematocrit, mean corpuscular volume, mean corpuscular $\mathrm{Hb}$ and mean corpuscular $\mathrm{Hb}$ concentration) were determined by a CELL-DYN 3700 autoanalyser (Abbott Diagnostics Europe, Wiesbaden, Germany). The CV for all automated measurements were below $5 \%$.

\section{Plasma quercetin and its metabolites}

Analyses of plasma concentrations of quercetin and of the monomethylated derivatives isorhamnetin (3'-O-methyl quercetin) and tamarixetin ( $4^{\prime}-O-$ methyl quercetin) were performed by HPLC with fluorescence detection as described previously ${ }^{(12)}$. All samples were treated enzymatically with $\beta$-glucuronidase/sulfatase type $\mathrm{H}-2$ (crude enzyme extract from Helix pomatia; Sigma-Aldrich AG, Taufkirchen, Germany) before the extraction of the flavonols. Authentic flavonols (Carl Roth GmbH, Karlsruhe, Germany) were used as external standards. The limit of detection was $5-7 \mathrm{nmol} / \mathrm{l}$. Inter-analysis and inter-day variances were within 5\%.

\section{Biokinetic calculations}

Plasma flavonol concentrations obtained at different time points (T30-T1440) were corrected for individual baseline flavonol concentrations (T0). Maximum plasma concentrations $\left(c_{\max }\right)$ and times to achieve maximum plasma concentrations $\left(t_{\max }\right)$ were obtained directly by the visual inspection of each participant's plasma concentration time profile. The area under the plasma concentration-time curve (AUC) was determined according to the linear trapezoidal rule.

\section{Statistical analysis}

Statistical analyses were performed using the SPSS statistical software package (version 17; SPSS, Inc., Chicago, IL, USA). All data were log-transformed before statistical analyses. The differences between the mean values of $c_{\max }, t_{\max }$ and AUC were analysed with the use of the paired Student's $t$ test. Changes between the baseline $(0 \mathrm{~min})$ and the following time points among the two treatment groups were tested for significance by repeated-measures ANOVA. Differences were considered significant at $P<0 \cdot 05$. Unless otherwise indicated, the results are reported as mean values with their standard errors.

\section{Results}

\section{Control variables}

No adverse effects of the quercetin intakes were reported. The dietary records did not show significant differences between the two treatment groups in mean daily intakes of energy $(9 \cdot 1 \mathrm{MJ} / \mathrm{d})$, protein $(14.8 \%$ of energy intake), carbohydrates ( $46.6 \%$ of energy intake), fat $(37.5 \%$ of energy intake), SFA and unsaturated fatty acid, cholesterol, antioxidants (vitamin E, $1.4 \mathrm{mg} / \mathrm{MJ}$; vitamin C, $15.6 \mathrm{mg} / \mathrm{MJ}$ ) or dietary fibre before the treatment (data not shown).

Fasting serum lipids, glucose, biomarkers of liver and kidney function and haematological parameters were all within normal ranges, and no significant differences were observed between the treatment groups (data not shown).

\section{Quercetin content of the study products}

Based on individual component analysis, quercetin-enriched cereal bars contained $67 \mathrm{mg}$ quercetin equivalents $/ 25 \mathrm{~g}$ (containing $93.3 \%$ quercetin aglycone plus $6.7 \%$ quercetin- $4^{\prime}$ glucoside). Thus, the participants ingested a total dose of $134 \mathrm{mg}$ quercetin equivalents (two cereal bars). The quercetin capsules contained $22 \mathrm{mg}$ quercetin aglycone per capsule leading to a total intake of $132 \mathrm{mg}$ quercetin aglycone (six capsules).

\section{Plasma quercetin}

The group mean plasma quercetin concentration-time curve for the six women after consuming a single dose of approximately $130 \mathrm{mg}$ quercetin from quercetin-enriched cereal bars and quercetin capsules, respectively, is shown in Fig. 1. In all participants, plasma quercetin increased $30 \mathrm{~min}$ following oral administration. This is consistent with in vitro digestion experiments, in which capsules and cereal bars were completely dispersed within the first digestion media simulating a fasting stomach fluid (data not shown).

The plasma quercetin concentration was consistently higher after the consumption of the quercetin-enriched cereal bars compared with quercetin intake from capsules. There was a high inter-individual variation in plasma concentration time profiles among the participants (Fig. 1). The participants who were the highest and the lowest quercetin absorbers after ingestion of the quercetin-enriched cereal bars were also the highest and the lowest absorbers after the intake of the quercetin capsules (data not shown).

Table 2 shows a summary of the results of the plasma kinetic analyses. Significant differences in the biokinetic parameters between the two treatments existed with respect to AUC and $c_{\max }$. The systemic availability as determined by comparing the plasma concentration-time curves of quercetin was found to be five times and $c_{\max }$ six times higher after ingestion of $130 \mathrm{mg}$ quercetin by quercetin-enriched cereal 


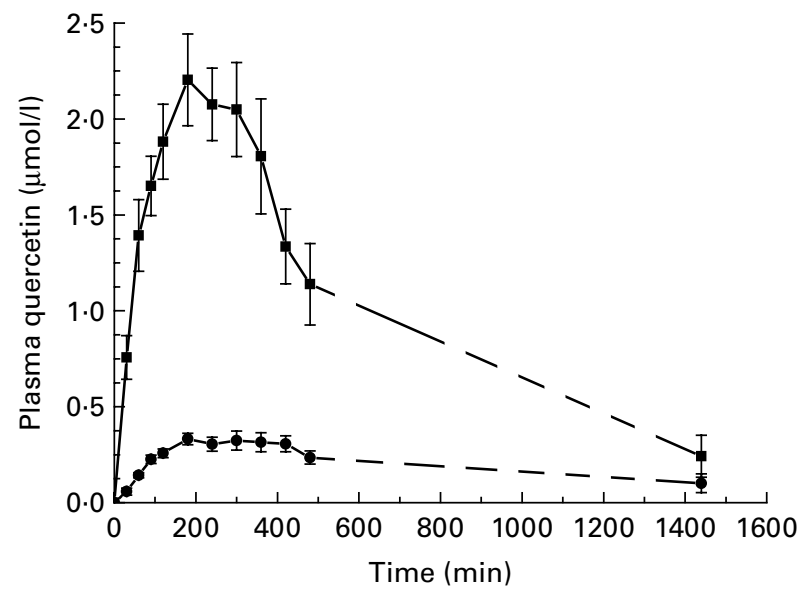

Fig. 1. Plasma appearance and disappearance curves of quercetin after the ingestion of quercetin from quercetin-enriched cereal bars ( $\square$ ) and quercetin powder-filled hard capsules ( circle; $n 6)$. Values are means, with their standard errors represented by vertical bars. Plasma concentrations differ significantly over time between the two treatment groups $(P<0.001$ for time effect and $P<0.001$ for time $\times$ treatment interaction in repeated-measures ANOVA). The last two data points are connected by a dashed line, because elimination processes during this time cannot be reliably determined.

bars than after ingestion of quercetin powder-filled capsules. In contrast, $t_{\max }$ did not differ significantly between the two treatments. Inter-individual variability of $c_{\max }$ values for plasma quercetin was $24.6 \%$ (cereal bars) and $25.0 \%$ (capsules).

\section{Plasma isorhamnetin and tamarixetin}

Following supplementation with quercetin, low amounts of isorhamnetin and tamarixetin were also apparent (Figs. 2 and 3). The plasma concentrations of isorhamnetin and tamarixetin were consistently higher after the consumption of the quercetin-enriched cereal bars compared with quercetin intake from capsules. There also was a high inter-individual variation in plasma concentration time profiles among the participants (Figs. 2 and 3). In all participants, tamarixetin was not detected in fasting plasma samples at baseline. The $c_{\max }$ values for isorhamnetin and tamarixetin were four and nine times higher after the ingestion of quercetin by quercetin-enriched cereal bars than after the ingestion of quercetin capsules (Table 2 ). In contrast, $t_{\max }$ values did not differ significantly between the two treatments (Table 2).

We did not calculate the AUC for isorhamnetin and tamarixetin because of the low and irregular plasma appearance and disappearance curves that made a reliable determination of the parameter impossible. Based on the $c_{\max }$ values, the monomethylated derivatives of quercetin (isorhamnetin and tamarixetin) accounted for about $14 \%$ (cereal bars) and 16\% (capsules) of total plasma flavonols $(=$ sum of quercetin $(\mu \mathrm{mol} / \mathrm{l})+$ isorhamnetin $(\mu \mathrm{mol} / \mathrm{l})+$ tamarixetin $(\mu \mathrm{mol} / \mathrm{l}))$.

\section{Discussion}

The aim of the present pilot study was to investigate the systemic availability of quercetin from cereal bars enriched with quercetin derived from onion skin extract and from powder-filled hard capsules, both applying equivalent amounts of quercetin (approximately $130 \mathrm{mg}$ ). We used this moderate supranutritional but non-pharmacological dose of quercetin, since our data should provide a rational basis for the development of functional foods. The quercetin dose represented ten- to fifteen-fold of the estimated daily quercetin intake in Germany of about $10 \mathrm{mg}^{(13,14)}$. Our data indicate that onion-derived quercetin from quercetin-enriched cereal bars is more effective at increasing plasma quercetin compared with quercetin from powder-filled hard capsules in a short-term protocol with a single-oral dose administration. This conclusion is based on the AUC values, which were significantly higher with quercetin from cereal bars than with quercetin from capsules.

Table 2. Biokinetic variables of plasma quercetin, isorhamnetin and tamarixetin concentrations in women after a single oral dose of quercetin from quercetin-enriched cereal bars and quercetin capsules (Mean values with their standard errors, $n 6$ )

\begin{tabular}{|c|c|c|c|c|c|}
\hline \multirow[b]{2}{*}{ Variable } & \multicolumn{2}{|c|}{$\begin{array}{l}\text { Quercetin-enriched } \\
\text { cereal bars }\end{array}$} & \multicolumn{2}{|c|}{ Quercetin capsules } & \multirow[b]{2}{*}{$P^{*}$} \\
\hline & Mean & SEM & Mean & $\overline{\text { SEM }}$ & \\
\hline \multicolumn{6}{|l|}{ Plasma quercetin } \\
\hline AUC $(\mu \mathrm{mol} / \mathrm{min}$ per I) & 1475.5 & $221 \cdot 7$ & $287 \cdot 8$ & $29 \cdot 3$ & $<0.001$ \\
\hline$c_{\max }(\mu \mathrm{mol} / \mathrm{l})$ & 2.48 & 0.25 & 0.40 & 0.04 & $<0.001$ \\
\hline$t_{\max }(\min )$ & 250 & 29 & 310 & 45 & 0.331 \\
\hline \multicolumn{6}{|l|}{ Plasma isorhamnetin } \\
\hline$c_{\max }(\mathrm{nmol} / \mathrm{l})$ & 194.2 & $22 \cdot 1$ & 54.4 & $13 \cdot 1$ & 0.010 \\
\hline$t_{\max }(\min )$ & 520 & 184 & 370 & 42 & 0.483 \\
\hline \multicolumn{6}{|l|}{ Plasma tamarixetin } \\
\hline$c_{\max }(\mathrm{nmol} / \mathrm{l})$ & $213 \cdot 1$ & $20 \cdot 6$ & $22 \cdot 9$ & $8 \cdot 2$ & $<0.001$ \\
\hline$t_{\max }(\min )$ & 340 & 33 & 160 & 55 & 0.124 \\
\hline
\end{tabular}




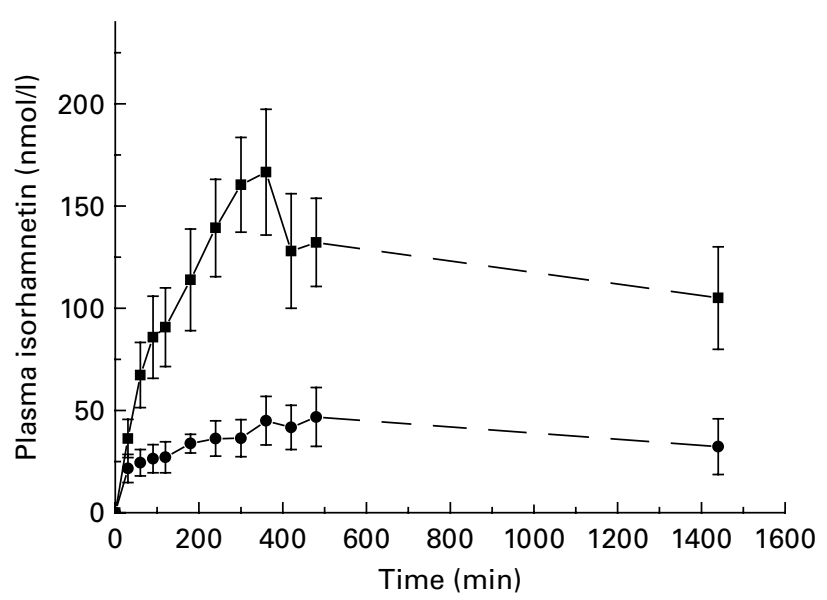

Fig. 2. Plasma appearance and disappearance curve for isorhamnetin after ingestion of quercetin from quercetin-enriched cereal bars $(\boldsymbol{\square})$ and quercetin powder-filled hard capsules $(\bullet ; n 6)$. Values are means, with their standard errors represented by vertical bars. Plasma concentrations differ significantly over time between the two treatment groups $(P<0.001$ for time effect and $P<0.001$ for time $\times$ treatment interaction in repeatedmeasures ANOVA). The last two data points are connected by a dashed line, because elimination processes during this time cannot be reliably determined.

The plasma profiles of quercetin after the ingestion of $130 \mathrm{mg}$ quercetin from capsules were similar to our previous human study that investigated the kinetics of plasma quercetin from three different quercetin dosages (50, 100 and $150 \mathrm{mg}$ quercetin dihydrate $)^{(15)}$. In accordance with previous human studies, we observed a relatively high inter-individual variance in plasma concentration-time curves of quercetin, isorhamnetin and tamarixetin following supplementation with quercetin ${ }^{(15-18)}$, although our non-smoking female study population was very homogenous with respect to age, baseline plasma quercetin concentrations, body weight, BMI, fat mass and fat-free mass as well as lifestyle and physical activity. This variability in plasma kinetic profiles may be attributed to inter-individual differences in digestive transit time, preferential site of absorption and enteric bacterial metabolism of quercetin as reported in the literature for quercetin and other polyphenols ${ }^{(3,19,20)}$ as well as to the intrinsic solubilisation capacity of each individual.

The $c_{\max }$ values (Table 2 ) indicated that the gastrointestinal absorption rate of quercetin from quercetin-enriched cereal bars was higher than from quercetin powder-filled hard capsules, which were ingested in combination with quercetin-free cereal bars. One factor that could explain this finding might be the food matrix. There are a limited number of studies concerning the effect of food components on the absorption of quercetin. Piskula \& Terao ${ }^{(21)}$ found that the solubility of quercetin in vehicles (propylene glycol, water and $25 \%$ propylene glycol in water $(\mathrm{v} / \mathrm{v}))$ for administration to rats greatly affected the efficiency of quercetin absorption. Sri et $a l .{ }^{(22)}$ demonstrated that the bioavailability of quercetin can be enhanced by a factor of 3 when inclusion complexes are formed with hydroxyl propyl- $\beta$-cyclodextrin. Azuma et $a l .{ }^{(23)}$ indicated that a combination of lipids (phosphatidylcholine and soyabean oil) and emulsifiers (sucrose fatty ester) can elevate the absorption of quercetin aglycone in rat plasma.
In pigs, Lesser et al. ${ }^{(24)}$ showed that the oral bioavailability of quercetin aglycone from a test meal was enhanced by approximately $60 \%$ by the addition of $15 \%$ (w:w) fat to a low-fat standard pig diet. It was speculated that the enhancement of quercetin bioavailability may have been the result of improved solubility of the lipophilic quercetin aglycone in the intestinal tract and/or additional absorption of quercetin via the lymphatic system in the presence of fat. In humans, however, a typical Western-type diet is likely to contain sufficient dietary fat to facilitate the absorption of querce$\operatorname{tin}^{(25)}$. Thus, the solubility and distribution of quercetin in bulk foods may be key factors for the bioavailability, because quercetin aglycone is not freely soluble in water.

The fact that quercetin capsules were administered together with quercetin-free cereal bars indicates that not merely do the components of the cereal bar affect the absorption of quercetin, but that the manufacturing process of the quercetincontaining cereal bar might be a key factor in explaining the present findings. Intimate mixing of quercetin with the components (solid macroparticles) of the cereal bar resulted in a homogenous distribution of quercetin. The addition of a viscous binding matrix (glucose-fructose syrup and soft sugar) converted the whole mixture after cooling into a solid dispersion. Solid dispersions provide a large surface for compounds with low solubility and are utilised for pharmaceuticals to enhance bioavailability. In fact, the effective surface area is one of the important factors governing the dissolution rate of poorly soluble compounds. Solid dispersions are, in addition to micronisation and nanosizing technologies, a measure of enhancing the effective surface area available for dissolution ${ }^{(26)}$

On the other hand, the hard capsules were filled with a powder, rather than a solid dispersion, containing $25 \mathrm{mg}$ quercetin dihydrate, and also did not contain flavonoid-solubilising excipients, which might have helped in the dissolution of the

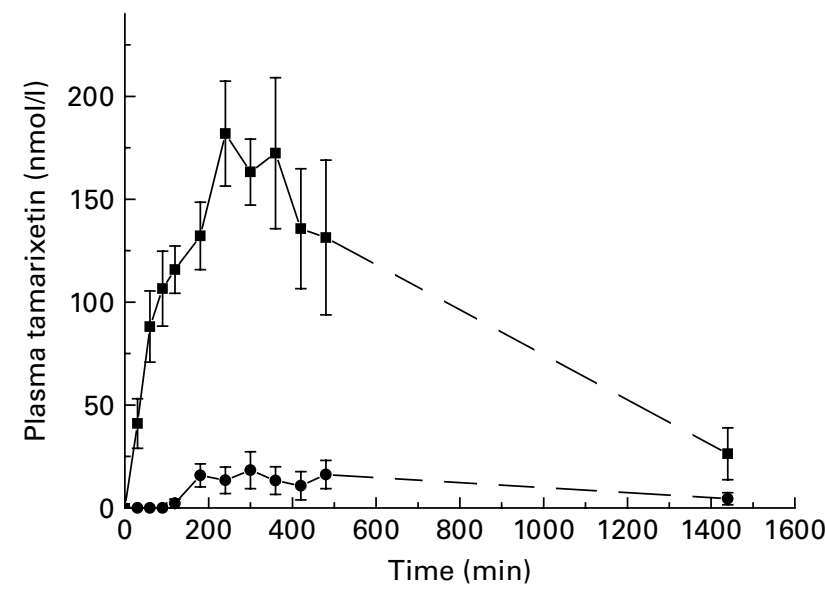

Fig. 3. Plasma appearance and disappearance curves for tamarixetin after ingestion of quercetin from quercetin-enriched cereal bars (ם) and quercetin powder-filled hard capsules $(0 ; n 6)$. Values are means, with their standard errors represented by vertical bars. Plasma concentrations differ significantly over time between the two treatment groups $(P<0.001$ for time effect and $P<0.001$ for time $\times$ treatment interaction in repeatedmeasures ANOVA). The last two data points are connected by a dashed line, because elimination processes during this time cannot be reliably determined. 
flavonoid from the dosage form. More advanced technologies, e.g. self-emulsifying systems in soft capsules and solid solutions/solid dispersions in glyceride-based matrices, might be developed to overcome the problem of poor bioavailability of quercetin powder from hard capsules.

After oral intake of quercetin from quercetin-enriched cereal bars and quercetin capsules, isorhamnetin and tamarixetin were also detected in human plasma. This finding is in accordance with our previous human kinetic study ${ }^{(15)}$ and has also been found in pigs ${ }^{(27,28)}$. However, in contrast to mice and rats, in which nearly one-half of the absorbed amount of quercetin is methylated to isorhamnetin ${ }^{(29-32)}$, the proportion of the methylated derivatives in human and pig plasma is rather low (approximately 15-20\% of total plasma flavonols). The finding that we detected the methylated quercetin metabolites in plasma samples shortly after the oral ingestion of quercetin indicates that these metabolites are derived from intestinal and/or hepatic metabolism by phase I enzymes, e.g. catechol-O-methyltransferase ${ }^{(33)}$, rather than being generated by microbial metabolisation.

To the best of our knowledge, the present study is the first to report that systemic availability of quercetin from quercetin-enriched cereal bars is significantly higher than that from quercetin capsules. The strengths of the present study are the randomised, blinded, diet-controlled cross-over design, the homogenous study population and the administration of equivalent amounts of quercetin (approximately $130 \mathrm{mg}$ ). However, the present study has some limitations. The quercetin-enriched cereal bars contained low amounts of quercetin- $4^{\prime}$-glucoside $(6.7 \%)$, whereas the quercetin capsules were free of any quercetin glucosides. The mechanisms of gastrointestinal absorption of the hydrophobic quercetin aglycone and of the glycosylated, more hydropholilic quercetin glucosides, differ ${ }^{(20,34)}$. Thus, the slightly different chemical composition of the quercetin profiles between cereal bar and capsule may have influenced the plasma appearance of quercetin. However, it seems unlikely that the low 'impurity' with quercetin- 4 -glucoside explains the big differences in the plasma concentration time profiles between the two application forms. More importantly, the physical form of the quercetin administered was not the same for the two treatments as the quercetin was isolated in two different ways. Quercetin dihydrate used for the hard capsule was isolated by crystallisation. In the cereal bars, quercetin was added in the form of an extract, i.e. an isolation process was used that includes an evaporation or sublimation process of the extraction solvent, which may lead to an amorphous structure. In turn, different solubilisation characteristics for quercetin may result ${ }^{(26)}$. In fact, an additional study showed that the systemic availability of approximately $100 \mathrm{mg}$ quercetin from hard capsules containing the onion skin extract was approximately two-fold higher than from quercetin dihydrate hard capsules (B Schulze, S Egert, S Wolffram, K Schwartz and $\mathrm{H}$ Stöckmann, unpublished results).

In conclusion, quercetin aglycone from quercetin-enriched cereal bars was more bioavailable than from quercetin hard capsules containing the flavonoid powder and solid fillers as excipients in human subjects. If the intake of quercetin and related flavonols can indeed be proven to reduce the risk of CVD, then the production of foods with a more highly bioavailable form of quercetin might become a realistic proposition. Modification of intestinal absorption by food factors may be a promising technique to control the physiological function and effectiveness of quercetin. However, the safety of a regular (long-term) intake of quercetin as an ingredient of functional foods also needs to be assessed.

\section{Acknowledgements}

We are indebted to Schwartauer-Werke GmbH \& Company KGaA (Dr Sebastian Portius) for the production of the cereal bars; to Rudolf Wild GmbH \& Company KG (Matthias Saß) for the supply of the onion skin extract and to A.C.T. FOODS GmbH, Kiel, Germany (Dr Heiko Stöckmann) for supporting the bioavailability testing of the onion skin extract. We thank Petra Schulz and Ute Hartung for valuable technical assistance. S. E., S. W., B. S., P. L., E. M. H., K. S., A. B.-W., G. R. and M. J. M. disclose that there are no conflicts of interest. B. A. is employed at Schwartauer-Werke GmbH \& Company KGaA. The present study was financially supported by the German Federal Ministry of Education and Research (BMBF 0313856A) within the project 'Functional Foods for Vascular Health - from Nutraceuticals to Personalised Diets' and Schwartauer-Werke GmbH \& Company KGaA. S. E., G. R. and M. J. M. designed the study; S. E. and M. J. M. conducted the study; P. L. produced the capsules; B. A. produced the cereal bars; A. B.-W. helped in collecting the clinical data; S. E., S. W., B. S., P. L., E. M. H. and K. S. analysed the data; S. E. performed the statistical analysis and wrote the first draft of the manuscript; S. W., B. S., P. L., K. S., G. R. and M. J. M. strongly contributed to the interpretation of the results. S. E. and M. J. M. had primary responsibility for the final content. All authors read and approved the final manuscript.

\section{References}

1. Erdman JW Jr, Balentine D, Arab L, et al. (2007) Flavonoids and heart health: proceedings of the ILSI North America Flavonoids Workshop, May 31-June 1, 2005, Washington, DC. J Nutr 137, 718S-737S

2. Graf BA, Milbury PE \& Blumberg JB (2005) Flavonols, flavones, flavanones, and human health: epidemiological evidence. J Med Food 8, 281-290.

3. Manach C, Williamson G, Morand C, et al. (2005) Bioavailability and bioefficacy of polyphenols in humans. I. Review of 97 bioavailability studies. Am J Clin Nutr 81, 230S-242S.

4. Scalbert A \& Williamson G (2000) Dietary intake and bioavailability of polyphenols. J Nutr 130, 2073S-2085S.

5. Harwood M, Danielewska-Nikiel B, Borzelleca JF, et al. (2007) A critical review of the data related to the safety of quercetin and lack of evidence of in vivo toxicity, including lack of genotoxic/carcinogenic properties. Food Chem Toxicol 45, 2179-2205.

6. Olthof MR, Hollman PC, Vree TB, et al. (2000) Bioavailabilities of quercetin-3-glucoside and quercetin- $4^{\prime}$-glucoside do not differ in humans. J Nutr 130, 1200-1203. 
7. Hollman PC, de Vries JH, van Leeuwen SD, et al. (1995) Absorption of dietary quercetin glycosides and quercetin in healthy ileostomy volunteers. Am J Clin Nutr 62, 1276-1282.

8. Wiczkowski W, Romaszko J, Bucinski A, et al. (2008) Quercetin from shallots (Allium cepa L. var. aggregatum) is more bioavailable than its glucosides. J Nutr 138, 885-888.

9. Egert S, Bosy-Westphal A, Seiberl J, et al. (2009) Quercetin reduces systolic blood pressure and plasma oxidised low-density lipoprotein concentrations in overweight subjects with a high-cardiovascular disease risk phenotype: a double-blinded, placebo-controlled cross-over study. Br J Nutr 102, 1065-1074.

10. Egert S, Boesch-Saadatmandi C, Wolffram S, et al. (2010) Serum lipid and blood pressure responses to quercetin vary in overweight patients by apolipoprotein E genotype. J Nutr 140, 278-284.

11. Bosy-Westphal A, Danielzik S, Becker C, et al. (2005) Need for optimal body composition data analysis using airdisplacement plethysmography in children and adolescents. J Nutr 135, 2257-2262.

12. Bieger J, Cermak R, Blank R, et al. (2008) Tissue distribution of quercetin in pigs after long-term dietary supplementation. J Nutr 138, 1417-1420.

13. Linseisen J, Radtke J \& Wolfram G (1997) Flavonoidzufuhr Erwachsener in einem bayrischen Teilkollektiv der Nationalen Verzehrsstudie (Flavonoid intake of adults in a Bavarian subgroup of the National Nutrition Survey). Z Ernabrungswiss 36, 403-412.

14. Böhm H, Boeing $\mathrm{H}$, Hempel J, et al. (1998) Flavonols, flavone and anthocyanins as natural antioxidants of food and their possible role in the prevention of chronic diseases. $Z$ Ernabrungswiss 37, 147-163.

15. Egert S, Wolffram S, Bosy-Westphal A, et al. (2008) Daily quercetin supplementation dose-dependently increases plasma quercetin concentrations in healthy humans. $J$ Nutr 138, 1615-1621.

16. Boyle SP, Dobson VL, Duthie SJ, et al. (2000) Absorption and DNA protective effects of flavonoid glycosides from an onion meal. Eur J Nutr 39, 213-223.

17. Graefe EU, Wittig J, Mueller S, et al. (2001) Pharmacokinetics and bioavailability of quercetin glycosides in humans. J Clin Pharmacol 41, 492-499.

18. Moon YJ, Wang L, Dicenzo R, et al. (2008) Quercetin pharmacokinetics in humans. Biopharm Drug Dispos 29, 205-217.

19. Williamson G \& Manach C (2005) Bioavailability and bioefficacy of polyphenols in humans. II. Review of 93 intervention studies. Am J Clin Nutr 81, 243S-255S.

20. Manach C, Scalbert A, Morand C, et al. (2004) Polyphenols: food sources and bioavailability. Am J Clin Nutr 79, $727-747$
21. Piskula MK \& Terao J (1998) Quercetin's solubility affects its accumulation in rat plasma after oral administration. J Agric Food Chem 46, 4313-4317.

22. Sri KV, Kondaiah A, Ratna JV, et al. (2007) Preparation and characterization of quercetin and rutin cyclodextrin inclusion complexes. Drug Dev Ind Pharm 33, 245-253.

23. Azuma K, Ippoushi K, Ito $\mathrm{H}$, et al. (2002) Combination of lipids and emulsifiers enhances the absorption of orally administered quercetin in rats. J Agric Food Chem 50, $1706-1712$.

24. Lesser S, Cermak R \& Wolffram S (2004) Bioavailability of quercetin in pigs is influenced by the dietary fat content. J Nutr 134, 1508-1511.

25. Lesser S, Cermak R \& Wolffram S (2006) The fatty acid pattern of dietary fat influences the oral bioavailability of the flavonol quercetin in pigs. Br J Nutr 96, 1047-1052.

26. Krishnaiah YSR (2010) Pharmaceutical technologies for enhancing oral bioavailability of poorly soluble drugs. J Bioequiv Bioavail 2, 28-36.

27. Ader P, Wessmann A \& Wolffram S (2000) Bioavailability and metabolism of the flavonol quercetin in the pig. Free Radic Biol Med 28, 1056-1067.

28. Cermak R, Landgraf S \& Wolffram S (2003) The bioavailability of quercetin in pigs depends on the glycoside moiety and on dietary factors. J Nutr 133, 2802-2807.

29. Boesch-Saadatmandi C, Egert S, Schrader C, et al. (2010) Effect of quercetin on paraoxonase 1 activity-studies in cultured cells, mice and humans. J Physiol Pharmacol 61, 99-105.

30. Boesch-Saadatmandi C, Wolffram S, Minihane AM, et al. (2009) Effect of apoE genotype and dietary quercetin on blood lipids and TNF-alpha levels in apoE3 and apoE4 targeted gene replacement mice. Br J Nutr 101, 1440-1443.

31. de Boer VC, Dihal AA, van der WH, et al. (2005) Tissue distribution of quercetin in rats and pigs. J Nutr $\mathbf{1 3 5}$, $1718-1725$.

32. Boesch-Saadatmandi C, Loboda A, Wagner AE, et al. (2010) Effect of quercetin and its metabolites isorhamnetin and quercetin-3-glucuronide on inflammatory gene expression: role of miR-155. J Nutr Biochem 22, 293-299.

33. Zhu BT, Ezell EL \& Liehr JG (1994) Catechol-O-methyltransferase-catalyzed rapid $O$-methylation of mutagenic flavonoids. Metabolic inactivation as a possible reason for their lack of carcinogenicity in vivo. J Biol Chem 269, 292-299.

34. Kottra G \& Daniel H (2007) Flavonoid glycosides are not transported by the human $\mathrm{Na}^{+} /$glucose transporter when expressed in Xenopus laevis oocytes, but effectively inhibit electrogenic glucose uptake. I Pharmacol Exp Ther 322, 829-835. 